\title{
Impact of Glasgow Coma Scale scores on unplanned intensive care unit readmissions among surgical patients
}

\author{
Tak Kyu $\mathrm{Oh}^{1}$, In-Ae Song ${ }^{1}$, Young-Tae Jeon ${ }^{1,2}$ \\ ${ }^{1}$ Department of Anesthesiology and Pain Medicine, Seoul National University Bundang Hospital, Seongnam-si, South Korea; ${ }^{2}$ Department of \\ Anesthesiology and Pain Medicine, College of Medicine, Seoul, South Korea \\ Contributions: (I) Conception and design: TK Oh; (II) Administrative support: YT Jeon; (III) Provision of study materials or patients: TK Oh; (IV) \\ Collection and assembly of data: IA Song, YT Jeon; (V) Data analysis and interpretation: TK Oh; (VI) Manuscript writing: All authors; (VII) Final \\ approval of manuscript: All authors. \\ Correspondence to: Young-Tae Jeon, MD, PhD. Department of Anesthesiology and Pain Medicine, Seoul National University Bundang Hospital, \\ Gumi-ro 173 Beon-gil, Bundang-gu, Seongnam 13620, South Korea. Email: ytjeon@snubh.org.
}

\begin{abstract}
Background: Physiological instability at discharge from intensive care units (ICU) is known to increase readmission rates among critically ill patients. However, associations between consciousness levels at discharge and readmission rates remain unclear. This study aimed to investigate the association between the Glasgow Coma Scale (GCS) score at discharge and unplanned ICU readmissions in surgical patients.

Methods: This retrospective cohort study in a single tertiary academic hospital analyzed the electronic health records of adults aged 18 years or older, who were discharged from the ICU between January 2012 and December 2018. The primary endpoint was unplanned readmission within 48 hours after discharge. Multivariable logistic regression analysis was performed.

Results: Among 9,512 patients, unplanned readmissions occurred in 161 (1.7\%). At discharge, GCS and verbal response scores of $\leq 13$ ( $v s . \geq 14$ ) were associated with 2.28 -fold higher unplanned readmissions within 48 hours [odds ratio (OR): $2.35,95 \%$ confidence interval (CI): $1.51-3.65, \mathrm{P}<0.001$ ]. Sensitivity analysis showed that verbal response scores of $\leq 4$ (vs. 5) at ICU discharge were associated with 2.21-fold higher unplanned readmissions within 48 hours (OR: 2.21, 95\% CI: 1.49-3.29, $\mathrm{P}<0.001$ ), whereas eye or motor responses at time of ICU discharge were not significantly associated with unplanned readmissions $(\mathrm{P}>0.05)$.

Conclusions: In this surgical ICU population cohort, GCS scores at ICU discharge were significantly associated with unplanned readmissions within 48 hours. This association was stronger with GCS scores of $\leq 13$ and with verbal response scores of $\leq 4$ at time of discharge. These findings suggest that surgical ICU patients with GCS scores of $\leq 13$ or verbal response scores of $\leq 4$ should be monitored carefully for discharge in order to avoid unplanned ICU readmissions.
\end{abstract}

Keywords: Critical care; Glasgow Coma Scale (GCS); intensive care units (ICU)

Submitted Jun 26, 2019. Accepted for publication Sep 23, 2019.

doi: $10.21037 /$ atm.2019.10.06

View this article at: http://dx.doi.org/10.21037/atm.2019.10.06

\section{Introduction}

The rate of intensive care unit (ICU) readmission after discharge is one of the key indicators of the quality of care and outcomes in critically ill patients treated in the ICU $(1,2)$. According to reports, ICU readmission rates range from $1.2 \%$ to $14.5 \%(3-6)$. ICU readmission is known to be associated with an increased length of hospital stay and mortality among critically ill patients $(3,4,7-10)$. Therefore, reducing these rates have considerable implications in terms of the improvement of hospital outcomes in critically ill patients, hospital resource utilization, and cost savings (11).

The Glasgow Coma Scale (GCS), which was introduced in 1974 by Teasdale and Jennett for the clinical assessment 
of unconscious patients, has been widely used as an important tool in the clinic for assessing the state of consciousness $(12,13)$. It has particular value in the assessment of trauma victims and critically ill patients (14), and is reportedly helpful in predicting in-ICU and hospital mortality in critically ill patients admitted to the ICU (15). Moreover, the GCS score at discharge is known to be predictive of 1-year outcomes in patients admitted to the ICU for severe traumatic brain injury. Since unstable vital signs at discharge is a well-known risk factor for ICU readmission (16), depressed states of consciousness at discharge may also be closely associated with ICU readmission. However, the association between the GCS score at discharge and ICU readmission remains unclear.

The present study therefore aimed to investigate the association between the GCS score at discharge and unplanned ICU readmissions within 48 hours among surgical patients. The present study presumed lower GCS scores at discharge to be associated with higher unplanned ICU readmission rates.

\section{Methods}

This retrospective cohort study in a single tertiary academic hospital was conducted with approval from the Institutional Review Board (IRB) of the Seoul National University Bundang Hospital (SNUBH) (IRB approval number: B-1904/534-103). Considering the retrospective design of this study, the requirement for informed consent was waived by the IRB.

\section{Data source and study population}

The data for the present study was obtained from the electronic health records in the Bundang Hospital Electronic System for Total Care (BESTCare) at SNUBH (17). The International Classification of Disease-10 (ICD-10) codes in the electronic health record system were used to calculate the Charlson comorbidity index of all patients. The study population comprised patients aged 18 years or older, who were discharged from the ICU at the SNUBH between January 2012 and December 2018. Patients transferred directly to another hospital after discharge from the ICU at SNUBH, those who could not be assessed for the GCS score owing to in-situ tracheostomy tubes at discharge, and those with incomplete or missing GCS scores or other medical records were excluded from the final analysis. During the studying period, discharge of surgical ICU patients was determined through discussion between certified intensivists and surgeons. Discharge criteria were not strictly consistent since the number of patients who needed to be admitted to the ICU in our hospital were based on available resources, and varied depending on the season, day of the week, and year.

\section{GCS score at discharge from the ICU (main independent variable)}

In SNUBH, all registered nurses were educated to assess and measure the GCS of ICU patients. The GCS score of patients at discharge from the ICU was defined as the score measured within at least 8 hours from discharge. In cases where GCS scores were measured twice or more within 8 hours, the score measured closest to the time of discharge was selected as the GCS score. The GCS score was calculated in the traditional manner as a sum of eye opening [1-4], verbal [1-5], and motor [1-6] response scores, with a range of 3-15 (12).

\section{Unplanned ICU readmission within 48 hours (dependent variable)}

Unplanned ICU readmission within 48 hours was defined as unplanned readmission to the ICU within 48 hours of being transferred to the general ward. Cases of ICU readmission within 48 hours after repeated surgery were classified as planned admission, and only direct unplanned readmissions to the ICU was considered as an unplanned ICU readmission.

\section{Potential confounders}

The data on the covariates collected for the study included the following: (I) physical characteristics (sex, age, and body mass index), (II) socioeconomic status related information at time of ICU admission (insurance type: national health insurance program versus medical aid beneficiary program, highest educational attainment: less than high school, more than or equal to high school, less than college, more than or equal to college, occupation: office worker or professional, licensed job, house work, self-employed, student, military, laborer, or unemployed, marital status: never married, married or living with someone, divorced or separated, widowed), (III) Charlson comorbidity index, (IV) Acute Physiology and Chronic Health Evaluation (APACHE) II score, (V) length of ICU stay (hours), (VI) postoperative 
ICU admission, (VII) admitting department (general surgery, cardiac surgery, major vascular surgery, thoracic surgery, neurosurgery, or other surgical department), (VIII) ventilator care during ICU stay (no ventilator care during ICU stay, ventilator care $\leq 24$ hours, ventilator care $>24$ hours), and (IX) year of discharge from the ICU (2012-2014, 2015-2016, or 2017-2018). Body mass index was calculated using the height $(\mathrm{cm})$ and weight $(\mathrm{kg})$ measured at ICU admission. In instances where height or weight could not be measured due to patient inability to stand, heights were determined through patient or guardian interviews and weights were measured using ICU beds capable of registering the weight of a lying patient. In our insurance system, patients with low income are listed in the medical aid program, and most hospital charges for these patients are paid for by the government. For patients in the national health insurance program, the government covers approximately two-thirds of overall hospital charges. Furthermore, the patient cohort was divided based on the year of ICU treatment into three groups (2012-2014, 20152016, and 2017-2018), since the overall mortality in ICU patients has been shown to be different across years (18).

\section{Statistical analysis}

The baseline characteristics of patients included in the study have been presented as the mean with standard deviations (SD) or as numbers with percentages. Initially, the restricted cubic spline was used to present the log odds of unplanned ICU readmissions within 48 hours, according to the GCS score [3-15] at discharge from the ICU. The cut-off value of the GCS score at discharge from the ICU was set to 14 and the patients were divided into 2 groups with GCS scores of $\leq 13$ and 14-15; This was done because the slope of the log odds of unplanned ICU readmissions within 48 hours in the restricted cubic spline was steep between scores of 15 and 14, but became gentle at a score of $<14$ (Figure S1).

Uni- and multi-variable logistic regression analyses were performed for unplanned ICU readmissions within 48 hours of discharge, based on the GCS score at discharge from the ICU. In this multivariable model, all covariates, namely, age, sex, body mass index, socioeconomic information (insurance type, occupation, marital status, and highest education level), APACHE II, Charlson comorbidity index, length of ICU stay, postoperative ICU admission, admitting department, ventilator care during ICU stay, and year of discharge from the ICU, were used for adjustment.
To avoid multi-collinearity of the main independent variable, the GCS score at discharge from the ICU, and the continuous and categorical variables (in the GCS score $\leq 13$ and $14-15$ groups) were included in separate multivariable models. Additionally, sensitivity analysis was performed to investigate the association between the 3 components of the GCS (eye, verbal, and motor responses) and unplanned ICU readmissions within $48 \mathrm{hrs}$. All results of the multivariable model have been presented as odds ratios (OR) with $95 \%$ confidence intervals (CI); the goodness of fit of each model was analyzed using the Hosmer and Lemeshow test. Additionally, the ORs with 95\% CIs that included all covariates have been presented in a forest plot. Finally, receiver operating characteristic (ROC) analysis was performed to determine the predictability of GCS, eye, verbal, and motor responses for unplanned readmission within 48 hours. Results of ROC analysis were represented as areas under the curve (AUCs) with 95\% CIs, and the Delong test was used to statistically compare AUC measures (19). All analyses were performed using the $\mathrm{R}$ software package, version 3.6.1 (R Foundation for Statistical Computing, Vienna, Austria) and $\mathrm{P}<0.05$ was considered to be statistically significant.

\section{Results}

A total of 11,552 patients were discharged from the ICU between January 2012 and December 2018. Among them, 712 cases repeatedly admitted twice or more were excluded from the analysis; therefore, 10,840 patients admitted to the ICU for the first time were selected for analysis. Among the 10,840 patients, 1,328 were excluded from the analysis. They included: (I) patients who were transferred directly to another hospital after discharge from the ICU ( $\mathrm{n}=114)$, (II) patients who were discharged with in-situ tracheostomy tubes $(\mathrm{n}=464)$, (III) patients with incomplete or missing GCS-related medical records at ICU discharge $(\mathrm{n}=358)$, and (IV) patients with missing or incomplete other medical records $(n=392)$. Finally, a total of 9,512 patients were included in the analysis, among whom, unplanned ICU readmissions within 48 hours was noted in $161(1.7 \%)$ patients (Figure 1). The baseline characteristics of the patients included in the final analysis are shown in Table 1. Among these patients, the mean (SD) GCS score at discharge from the ICU was 14.4 (1.0). The main diagnoses for ICU readmission within 48 hours among surgical patients are presented in Table 2. The most common main diagnoses at the time of unplanned ICU readmission within 


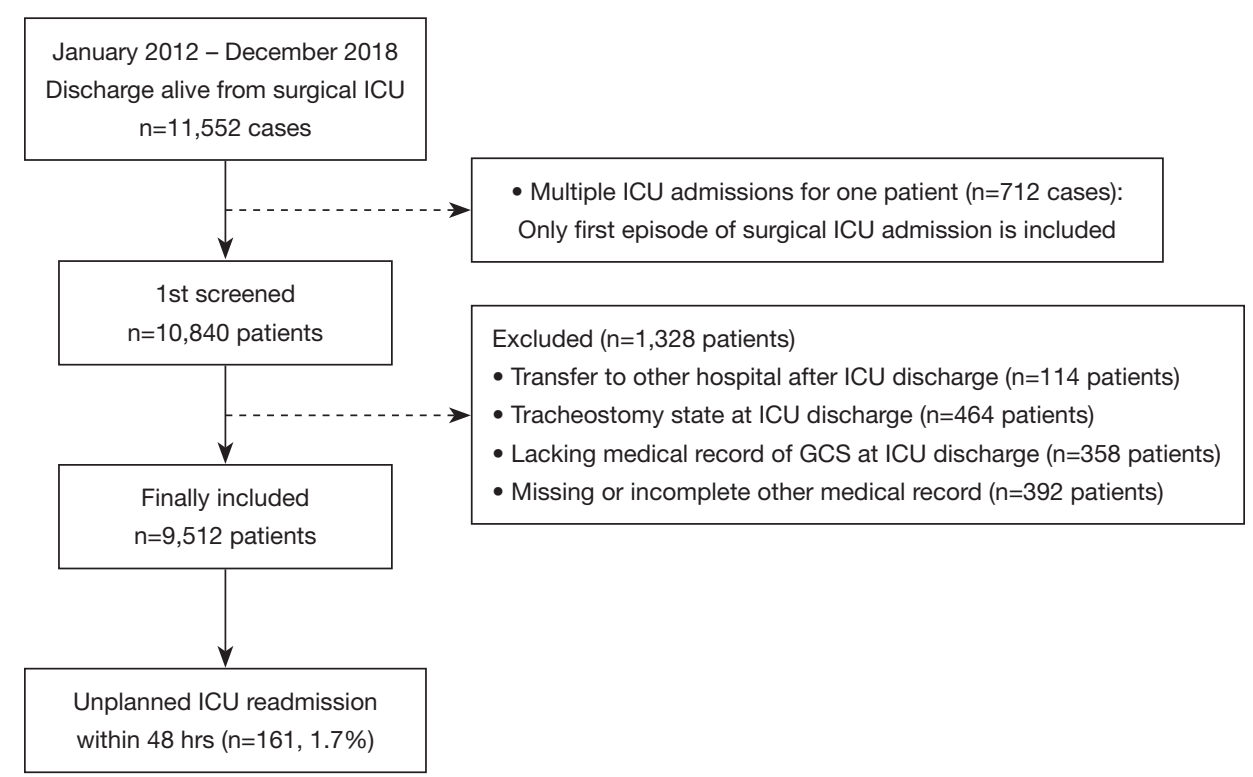

Figure 1 Flow chart depicting patient selection. ICU, intensive care unit; GCS, Glasgow Coma Scale.

48 hours was respiratory failure or insufficiency (51/161, $31.7 \%)$, followed by sepsis or septic shock (28/161, 17.4\%), and neurologic events (24/161, 14.9\%).

\section{Unplanned ICU readmissions within 48 hours}

The results of the logistic regression analysis before and after multivariable adjustment for unplanned ICU readmissions within 48 hours are shown in Table 3. In the covariates-adjusted multivariable model, an increase in the GCS score by 1 point at discharge from the ICU resulted in a $12 \%$ decrease in unplanned ICU readmissions within 48 hours (OR: 0.85, 95\% CI: 0.75-0.95; P=0.005; model 1). Moreover, patients with GCS scores of $\leq 13$ at discharge from the ICU experienced a 2.35 -fold increase in unplanned ICU readmissions within 48 hours as compared to those with scores of $\geq 14$ (OR: 2.35; 95\% CI: $1.51-3.65$; $\mathrm{P}<0.001$; model 2; Figure 2). On sensitivity analysis, patients with verbal response scores of $\leq 4$ at discharge demonstrated a 2.21-fold increase in unplanned ICU readmissions within 48 hours compared to those with scores of 5 (OR: 2.21; $95 \%$ CI: 1.49-3.29; $\mathrm{P}<0.001$; model 3); eye or motor responses at discharge were not significantly associated with unplanned ICU readmissions within 48 hours $(\mathrm{P}>0.05)$.

\section{ROC curve analysis}

The results of ROC analysis for unplanned readmission within 48 hours are shown in Table 4. The AUC of GCS at ICU discharge was 0.59 (95\% CI: 0.58-0.60), and were significantly higher than that of eye response (AUC: 0.53 , 95\% CI: $0.52-0.54$ ) or motor response (AUC: 0.51, 95\% CI: 0.50-0.52) using the Delong test. However, the AUC of verbal response was 0.60 (95\% CI: $0.59-0.61$ ) and was not statistically different from the GCS at ICU discharge $(\mathrm{Z}=0.352, \mathrm{P}=0.725)$.

\section{Discussion}

The findings in this cohort show that in surgical patients, the GCS score at the time of discharge from the ICU is associated with unplanned ICU readmissions within 48 hours. In particular, GCS scores of $\leq 13$ increase unplanned ICU readmission rates; among the components of the GCS, namely, eye, verbal, and motor responses, the verbal response at the time of discharge from the ICU was the factor most closely associated with unplanned ICU readmissions. Our study suggested that surgical ICU patients with GCS scores of $\leq 13$ or verbal response scores of $\leq 4$ should be carefully considered for ICU discharge in order to avoid unplanned ICU readmission. Additionally, if patients with GCS scores of $\leq 13$ or verbal response scores of $\leq 4$ are discharged from the ICU, they should be closely monitored in the general ward. Since ICU utilization is a limited high cost resource in many hospitals (20), our findings provide information that is useful for determining 
Table 1 Baseline characteristics of patients who discharged surgical ICU from 2012 to 2018

\begin{tabular}{|c|c|}
\hline Variable & Total $(n=9,512)$ \\
\hline Sex: male & $4,937(51.9)$ \\
\hline Age, year & $60.4 \pm 15.8$ \\
\hline Body mass index, $\mathrm{kg} \cdot \mathrm{m}^{-2}$ & $24.0 \pm 3.7$ \\
\hline \multicolumn{2}{|l|}{ Insurance type $^{a}$} \\
\hline National health insurance program & $9,191(96.6)$ \\
\hline Medical aid beneficiary program & $321(3.4)$ \\
\hline \multicolumn{2}{|l|}{ Highest educational attainment } \\
\hline Lower than high school & $2,795(29.4)$ \\
\hline $\begin{array}{l}\text { More than or equal to high school, lower } \\
\text { than college }\end{array}$ & $2,601(27.3)$ \\
\hline More than or equal to college & $4,116(43.3)$ \\
\hline \multicolumn{2}{|l|}{ Occupation at ICU admission } \\
\hline Office worker & $2,344(24.6)$ \\
\hline Professional (licensed job) & $486(5.1)$ \\
\hline House work & $2,335(24.5)$ \\
\hline Self-employed & $997(10.5)$ \\
\hline Student, military, or laborer & $940(9.9)$ \\
\hline Unemployed & $2,410(25.3)$ \\
\hline \multicolumn{2}{|l|}{ Marital status at ICU admission } \\
\hline Never married & $1,959(20.6)$ \\
\hline Married or living with someone & $6,546(68.8)$ \\
\hline Divorced or separated & $300(3.2)$ \\
\hline Widowed & $707(7.4)$ \\
\hline Charlson comorbidity index & $0.5 \pm 1.1$ \\
\hline APACHE II & $22.3 \pm 7.5$ \\
\hline Length of ICU stay, h & $46.4 \pm 277.2$ \\
\hline Postoperative ICU admission & $7,730(81.3)$ \\
\hline \multicolumn{2}{|l|}{ Admitting department } \\
\hline General surgery & $1,142(12.0)$ \\
\hline Cardiac surgery & $880(9.3)$ \\
\hline Major vascular surgery & $1,180(12.4)$ \\
\hline Thoracic surgery & $328(3.4)$ \\
\hline Neurosurgery & $4,049(42.6)$ \\
\hline Other surgical departments & $1,933(20.3)$ \\
\hline
\end{tabular}

Table 1 (continued)
Table 1 (continued)

\begin{tabular}{|c|c|}
\hline Variable & Total $(n=9,512)$ \\
\hline \multicolumn{2}{|l|}{ Ventilator care } \\
\hline No ventilator care during ICU stay & $6,806(71.6)$ \\
\hline Ventilator care $\leq 24 \mathrm{~h}$ & $1,342(14.1)$ \\
\hline Ventilator care $>24 \mathrm{~h}$ & $1,364(14.3)$ \\
\hline \multicolumn{2}{|l|}{ Year of ICU discharge } \\
\hline 2012-2014 & $4,066(42.7)$ \\
\hline 2015-2016 & $2,464(25.9)$ \\
\hline 2017-2018 & $2,982(31.3)$ \\
\hline Glasgow Coma Scale at ICU discharge & $14.4 \pm 1.0$ \\
\hline Eye response & $3.7 \pm 0.5$ \\
\hline$\leq 3$ & $2,882(30.3)$ \\
\hline Verbal response & $4.7 \pm 0.6$ \\
\hline$\leq 4$ & $1,789(18.8)$ \\
\hline Motor response & $5.9 \pm 0.3$ \\
\hline$\leq 5$ & $9,276(97.5)$ \\
\hline Glasgow Coma Scale at discharge $\leq 13$ & $947(10.0)$ \\
\hline \multicolumn{2}{|c|}{$\begin{array}{l}\text { Presented as number (percentage) or mean } \pm \text { standard } \\
\text { deviation. }{ }^{2} \text {, the patients in the medical aid beneficiary program } \\
\text { are those who are classified to have low income, and most of } \\
\text { their hospital charges are paid by the government. Meanwhile, } \\
\text { for the patients in the national health insurance program, } \\
\text { approximately two-thirds of their hospital charges are covered } \\
\text { by the government. SD, standard deviation; APACHE, acute } \\
\text { physiology and chronic health evaluation. }\end{array}$} \\
\hline
\end{tabular}

Table 2 Main diagnosis at ICU readmission within $48 \mathrm{~h}$ among surgical patients

\begin{tabular}{lc}
\hline Main diagnosis & Total 161 cases (\%) \\
\hline Respiratory failure or insufficiency & $51(31.7)$ \\
Acute kidney injury & $7(4.3)$ \\
Bleeding & $12(7.5)$ \\
Neurologic event & $24(14.9)$ \\
Arrhythmia & $8(5.0)$ \\
Cardiogenic shock & $22(13.7)$ \\
Cardiac arrest & $4(2.5)$ \\
Sepsis or septic shock & $28(17.4)$ \\
Others & $5(3.1)$ \\
\hline
\end{tabular}

ICU, intensive care unit. 
Table 3 Logistic regression analysis for unplanned ICU readmission within $48 \mathrm{~h}$

\begin{tabular}{|c|c|c|}
\hline Variable & OR $(95 \% \mathrm{Cl})$ & $P$ value \\
\hline GCS at discharge (per 1 point) & $0.83(0.75-0.92)$ & $<0.001$ \\
\hline$\leq 13$ (vs. $\geq 14)$ & $2.38(1.61-3.51)$ & $<0.001$ \\
\hline \multicolumn{3}{|l|}{ Covariate-adjusted $^{*}$} \\
\hline$\leq 13$ (vs. $\geq 14 ;$ model 2 ) & $2.35(1.51-3.65)$ & $<0.001$ \\
\hline \multicolumn{3}{|l|}{ Sensitivity analysis (model 3 ) } \\
\hline Eye response: $\leq 3$ (vs. 4) & $1.26(0.77-2.04)$ & 0.358 \\
\hline Verbal response: $\leq 4$ (vs. 5) & $2.21(1.49-3.29)$ & $<0.001$ \\
\hline
\end{tabular}

*, all covariates (age, sex, BMI, Insurance type, marital status, occupation, highest educational attainment, APACHE II, Charlson comorbidity index, length of ICU stay, postoperative ICU admission, admitting department, type of surgery, ventilator care, and year of ICU discharge) were included in multivariable model for adjustment. Hosmer and Lemeshow statistics (Chi-square: 10.6, $\mathrm{P}=0.221$ in model 1, Chi-square: $11.26, P=0.188$ in model 2, Chi-square: $11.62, P=0.169$ in model 3). OR, odds ratio; $\mathrm{Cl}$, confidence interval; APACHE, Acute Physiology and Chronic Health Evaluation.

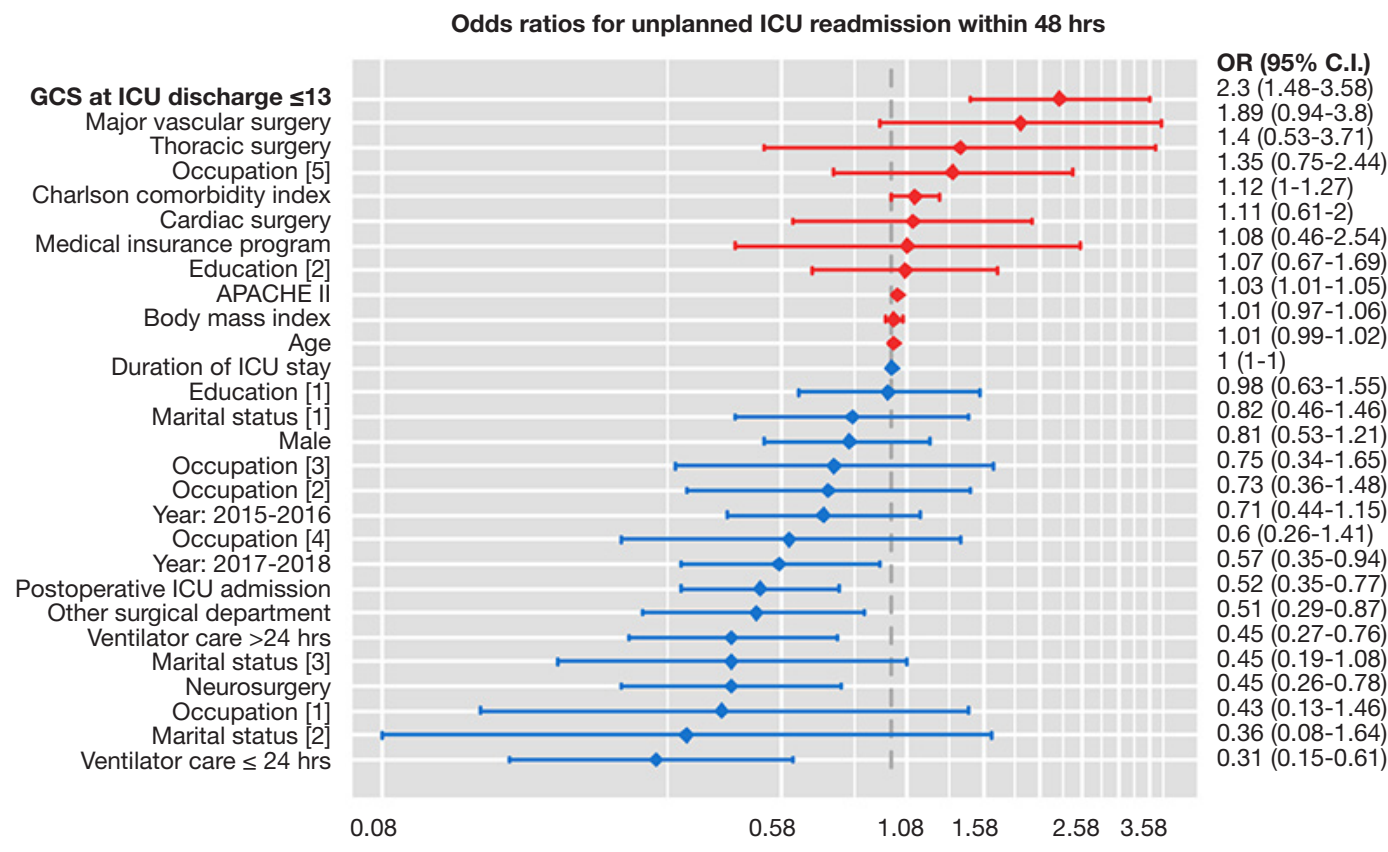

Figure 2 Odds ratios for unplanned readmissions within 48 hours after discharge from the ICU. Occupation [1]: licensed job vs. office worker; occupation [2]: house work vs. office worker; occupation [3]: self-employed vs. office worker; occupation [4]: student, military, or labourer $v s$. office worker; occupation [5]: unemployed $v$ s. office worker. Highest educational attainment [1]: more than or equal to high school. Lower than college vs. lower than high school; highest educational attainment [2]: more than or equal to college vs. lower than high school. Marital status [1]: married or living together vs. never married; marital status [2]: divorced or separated vs. never married; marital status [3]: widowed $v s$. never married. ICU, intensive care unit; APACHE, acute physiology and chronic health evaluation. 
Table 4 ROC analysis for unplanned readmission within $48 \mathrm{~h}$ among surgical ICU patients

\begin{tabular}{lc}
\hline Variable & $\begin{array}{c}\text { Area under curve }(95 \% \\
\text { Cl) }\end{array}$ \\
\hline Glasgow Coma Scale at ICU discharge [1] & $0.59(0.58-0.60)$ \\
Eye response at ICU discharge [2] & $0.53(0.52-0.54)$ \\
Motor response at ICU discharge [3] & $0.51(0.50-0.52)$ \\
Verbal response at ICU discharge [4] & $0.60(0.59-0.61)$
\end{tabular}

[1] vs. [2]: $Z=3.15, P=0.002$, [1] vs. [3]: $Z=0.352, P=0.725$, [1] vs. [4]: $Z=3.33, P<0.001$, [2] vs. [3]: $Z=2.27, P=0.023$, [2] vs. [4]: $Z=0.968, P=0.323$, [3] vs. [4]: $Z=4.10, P<0.001$. $R O C$, receiver operating characteristic; ICU, intensive care unit.

\section{ICU discharge criteria.}

In a previous cohort study, Lee et al. reported that the APACHE II score at discharge from the ICU was associated with readmission among surgical patients (21). A cohort study by Lin et al. also reported that a higher APACHE II score was associated with an increase in unplanned ICU readmissions within $48 \mathrm{~h}$ (22). Since the calculation of the APACHE II score considers the GCS (23), results from previous studies may be used as reference to interpret the findings of the present study $(21,22)$. However, the APACHE II score includes 11 other physiologic variables in addition to the GCS, and reflects the overall physiologic status instead of the levels of consciousness (23). Therefore, the present study is unique in that it analyzed the rates of readmission to the ICU among surgical patients with a focus on levels of consciousness, which were assessed by the GCS score at the time of discharge from the ICU.

Interestingly, in the present study, only verbal response at discharge showed significant association with unplanned ICU readmissions rate on sensitivity analysis. This is a novel finding among the studies on patients admitted to the ICU. A recent study on healthy volunteers (24) found that the human verbal intelligence is formed by the responsiveness of sensory and higher-order brain systems to external stimulation. From the perspective of cognitive neuroscience, this indicates that compared to eye opening and motor response, verbal communication or orientation, assessed by verbal responses in the GCS, may be used to assess higher-cortical brain function (25). Therefore, from the neurophysiologic perspective, critically ill patients who demonstrate perfect verbal response scores of 5 at discharge from the ICU, are highly likely to have achieved stable recovery.
The cut-off GCS score of $\leq 13$ at discharge from the ICU is also noteworthy. The present study established the cut-off point of 13 based on the slope of the restricted cubic spline between GCS scores and unplanned ICU readmission rates. Traditionally, GCS scores of 14-15 are considered to represent mild severity in patients with traumatic brain injury; in comparison, GCS scores of 13 and below represent moderate brain injury (26). Moreover, according to other reports, patients with blunt trauma and GCS scores of $\leq 13$ must be evaluated individually and closely monitored, since these patients may require emergent intubation (27). To date, there are no reports on the differences in prognosis among critically ill or surgical patients, based on cut-off values of the GCS score. The significant differences in clinical outcomes, based on a cutoff GCS score of $\leq 13$ at discharge from the ICU could have important implications.

Results of additional ROC analysis for unplanned ICU readmission within 48 hours used to determine the predictability of GCS scores in this study were also notable. Although the GCS scores or verbal scores at ICU discharge were significantly associated with unplanned ICU readmission, the AUCs were relatively poor (AUC $\leq 0.6)$. This indicated that GCS scores alone cannot be used as predictive tools for unplanned ICU readmission, and that GCS values at ICU discharge should be used as an additional tool for determining ICU discharge criteria.

The present study has some limitations. First, owing to the inherent limitation of using a retrospective cohort design, the presence of confounders could not be avoided. However, multivariable adjustment was employed. Second, since the study was conducted in a single tertiary academic hospital, the generalizability of the findings is limited. There is a possibility that our findings may not be applicable to other hospitals. Third, patients who had undergone tracheostomy and were unable to respond verbally were excluded from the analysis to allow an accurate assessment of the GCS scores. However, this exclusion may have confounded the results. Fourth, although ICU patient socioeconomic information including insurance type, occupation, marital status, and highest educational attainment were included as potential study confounders, they may still have influenced the overall study results. Fifth, although a previous study reported that GCS verbal scores could be calculated using motor and eye responses (28), we did not calculate this measure for patients that had in-situ tracheostomy tubes at discharge. This was due to the fact that while GCS was the main independent 
variable, we considered that the study homogeneity would be altered if this method of calculation was used (28). However, it could be argued that this may be considered a limitation of the study and as such we have included it in this section. Lastly, although the study included various confounders in the multivariable models, certain unmeasured confounders may have been present, which could have introduced bias.

In conclusion, the findings from the present study show that the GCS score at discharge from ICU is significantly associated with unplanned ICU readmission within 48 hours in the surgical ICU population. In particular, this association is more evident among patients with GCS scores of $\leq 13$ at discharge from the ICU, and those with verbal response scores of $\leq 4$. These findings suggest that surgical ICU patients with GCS scores of $\leq 13$ or verbal response scores of $\leq 4$ should be carefully considered for ICU discharge in order to prevent unplanned ICU readmission.

\section{Acknowledgments}

None.

\section{Footnote}

Conflicts of Interest: The authors have no conflicts of interest to declare.

Ethical Statement: The authors are accountable for all aspects of the work in ensuring that questions related to the accuracy or integrity of any part of the work are appropriately investigated and resolved. This study was approved by the Institutional Review Board of SNUBH (IRB approval number: B-1904/534-103) and waived the need for informed consent because of the retrospective study design.

\section{References}

1. Berenholtz SM, Dorman T, Ngo K, et al. Qualitative review of intensive care unit quality indicators. J Crit Care 2002;17:1-12.

2. Rhodes A, Moreno RP, Azoulay E, et al. Prospectively defined indicators to improve the safety and quality of care for critically ill patients: a report from the Task Force on Safety and Quality of the European Society of Intensive Care Medicine (ESICM). Intensive Care Med
2012;38:598-605.

3. Elliott M, Worrall-Carter L, Page K. Intensive care readmission: a contemporary review of the literature. Intensive Crit Care Nurs 2014;30:121-37.

4. Kramer AA, Higgins TL, Zimmerman JE. The association between ICU readmission rate and patient outcomes. Crit Care Med 2013;41:24-33.

5. Brown SE, Ratcliffe SJ, Kahn JM, et al. The epidemiology of intensive care unit readmissions in the United States. Am J Respir Crit Care Med 2012;185:955-64.

6. Al-Jaghbeer MJ, Tekwani SS, Gunn SR, et al. Incidence and Etiology of Potentially Preventable ICU Readmissions. Crit Care Med 2016;44:1704-9.

7. de Lima V, Bierrenbach AL, Alencar GP, et al. Increased risk of death and readmission after hospital discharge of critically ill patients in a developing country: a retrospective multicenter cohort study. Intensive Care Med 2018;44:1090-6.

8. Jeong BH, Na SJ, Lee DS, et al. Readmission and hospital mortality after ICU discharge of critically ill cancer patients. PLoS One 2019;14:e0211240.

9. Kareliusson F, De Geer L, Tibblin AO. Risk prediction of ICU readmission in a mixed surgical and medical population. J Intensive Care 2015;3:30.

10. Ponzoni CR, Correa TD, Filho RR, et al. Readmission to the Intensive Care Unit: Incidence, Risk Factors, Resource Use, and Outcomes. A Retrospective Cohort Study. Ann Am Thorac Soc 2017;14:1312-9.

11. Kramer AA, Higgins TL, Zimmerman JE. Intensive care unit readmissions in U.S. hospitals: patient characteristics, risk factors, and outcomes. Crit Care Med 2012;40:3-10.

12. Rowley G, Fielding K. Reliability and accuracy of the Glasgow Coma Scale with experienced and inexperienced users. Lancet 1991;337:535-8.

13. Teasdale G, Jennett B. Assessment of coma and impaired consciousness. A practical scale. Lancet 1974;2:81-4.

14. Sternbach GL. The Glasgow coma scale. The Journal of emergency medicine 2000;19:67-71.

15. Bastos PG, Sun X, Wagner DP, et al. Glasgow Coma Scale score in the evaluation of outcome in the intensive care unit: findings from the Acute Physiology and Chronic Health Evaluation III study. Crit Care Med 1993;21:1459-65.

16. Rosenberg AL, Watts C. Patients readmitted to ICUs* : a systematic review of risk factors and outcomes. Chest 2000;118:492-502.

17. Yoo S, Lee KH, Lee HJ, et al. Seoul National University Bundang Hospital's Electronic System for Total Care. 
Healthc Inform Res 2012;18:145-52.

18. Zimmerman JE, Kramer AA, Knaus WA. Changes in hospital mortality for United States intensive care unit admissions from 1988 to 2012. Crit Care 2013;17:R81.

19. DeLong ER, DeLong DM, Clarke-Pearson DL.

Comparing the areas under two or more correlated receiver operating characteristic curves: a nonparametric approach. Biometrics 1988;44:837-45.

20. Chang DW, Shapiro MF. Association Between Intensive Care Unit Utilization During Hospitalization and Costs, Use of Invasive Procedures, and Mortality. JAMA Intern Med 2016;176:1492-9.

21. Lee H, Lim CW, Hong HP, et al. Efficacy of the APACHE II score at ICU discharge in predicting postICU mortality and ICU readmission in critically ill surgical patients. Anaesth Intensive Care 2015;43:175-86.

22. Lin WT, Chen WL, Chao CM, et al. The outcomes and prognostic factors of the patients with unplanned intensive care unit readmissions. Medicine (Baltimore) 2018;97:e11124.

23. Knaus WA, Draper EA, Wagner DP, et al. APACHE II:

Cite this article as: Oh TK, Song IA, Jeon YT. Impact of Glasgow Coma Scale scores on unplanned intensive care unit readmissions among surgical patients. Ann Transl Med 2019;7(20):520. doi: 10.21037/atm.2019.10.06 a severity of disease classification system. Crit Care Med 1985;13:818-29.

24. Naci L, Haugg A, MacDonald A, et al. Functional diversity of brain networks supports consciousness and verbal intelligence. Sci Rep 2018;8:13259.

25. Luria AR. Higher cortical functions in man. Springer Science \& Business Media; 2012.

26. Mena JH, Sanchez AI, Rubiano AM, et al. Effect of the modified Glasgow Coma Scale score criteria for mild traumatic brain injury on mortality prediction: comparing classic and modified Glasgow Coma Scale score model scores of 13. J Trauma 2011;71:1185-92; discussion 1193.

27. Hsiao AK, Michelson SP, Hedges JR. Emergent intubation and CT scan pathology of blunt trauma patients with Glasgow Coma Scale scores of 3-13. Prehosp Disaster Med 1993;8:229-36.

28. Rutledge R, Lentz CW, Fakhry S, et al. Appropriate use of the Glasgow Coma Scale in intubated patients: a linear regression prediction of the Glasgow verbal score from the Glasgow eye and motor scores. J Trauma 1996;41:514-22. 

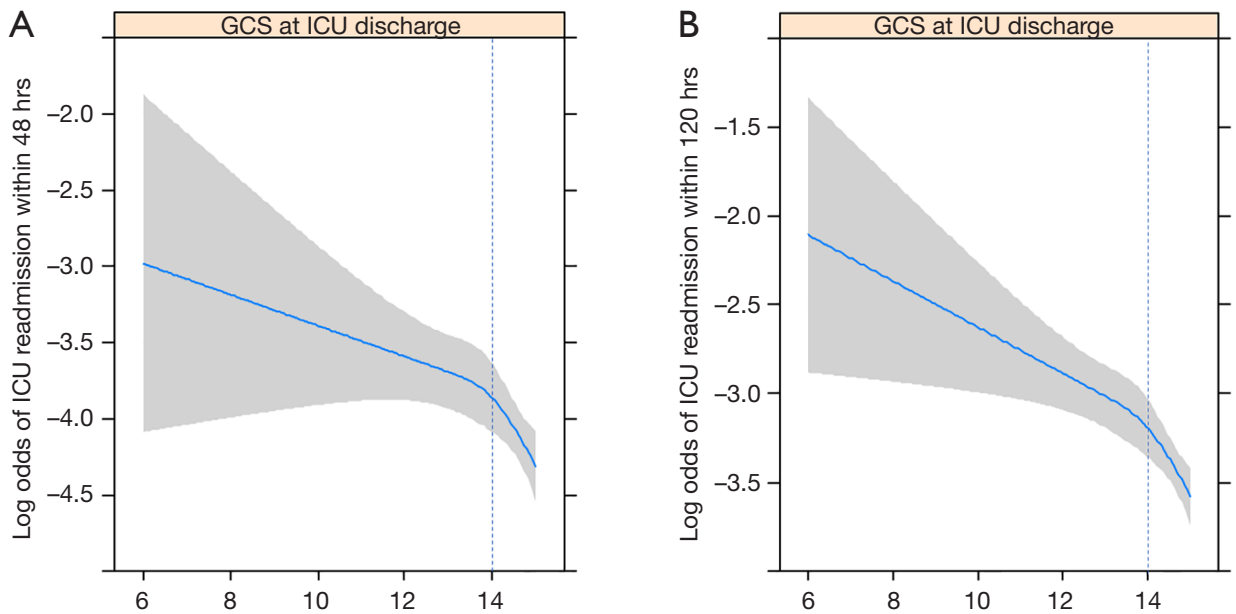

Figure S1 Log odds of ICU readmission within (A) 48 hours and (B) 120 hours after discharge from the ICU according to the GCS score at discharge. ICU, intensive care unit; GCS, Glasgow Coma Scale. 